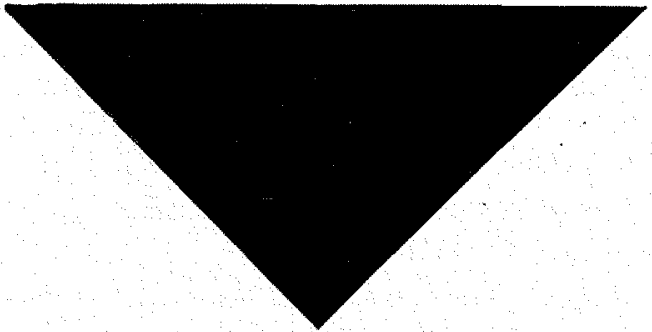

\section{DIFFRACTION DE \\ LA HOULE SUR DES OBSTACLES A PAROIS VERTICALES}

La solution analytique du problème de la diffraction de la houle par un obstacle à parois verticales n'est connue que pour des obstacles de formes simples : piles de section circulaire ou clliptique, jetées rectilignes, etc.

Dans le cas plus général où plusieurs obstacles de contours horizontaux quelconques sont placés dans la houle, il n'est pas possible d'aboutir a la solution complète uniquement par le calcul a la plume.

Depuis quelques années, gràce aux possibilités des ordinateurs électroniques, on peut résoudre par voie numérique un nombre beaucoup plus grand de cas pratiques.

La première partie de cet article expose la méthode employée pour calculer l'agitation et les efforts sur des structures placées dans la toule en théorie linéaire.

La seconde partie donne quelques résultats sous forme graphique pour les types de piles les plus courants.

\footnotetext{
* Ingénicurs au Centre de Recherches et d'Essais de Chatou
} (E.D.F.).

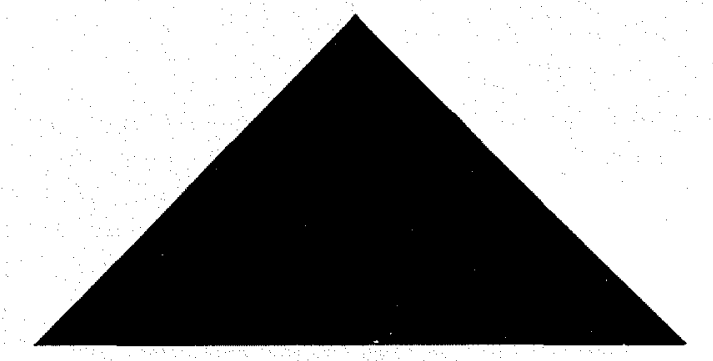

PAR A. DAUBERT * ET J.-C. LEBRETON *
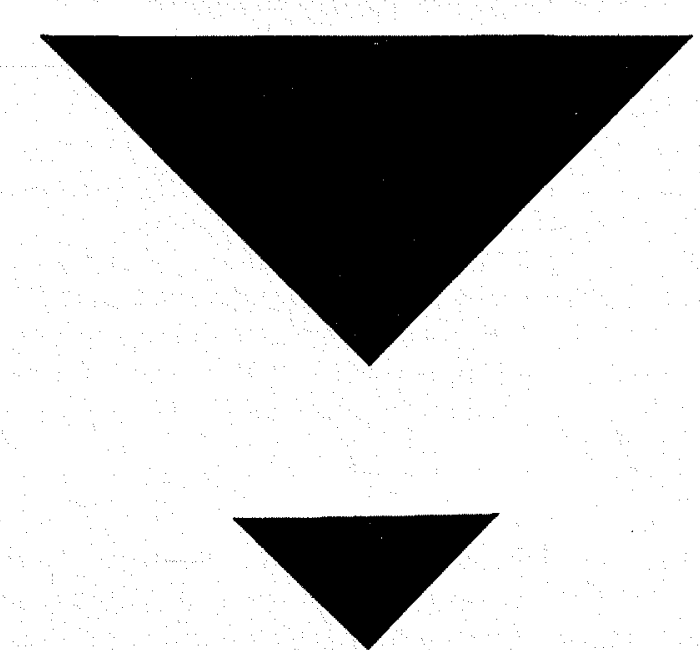

PREMIERE PARTIE

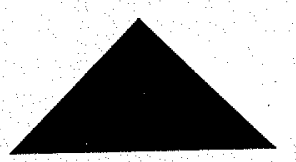

Introduction

Cette étude se propose de déterminer la déformation de la houle au voisinage de piles cylindriques à parois verticales.

La profondeur est supposée constante et les obstacles s'étendent de la surface libre jusqu'au fond du fluide.

Si tous les obstacles introduits dans la houle étaient «transparents» à la houle, celle-ci continuerait son chemin sans déformation. L'agitation, c'est-à-dire l'amplitude maximale, serait parfaitement connue. L'effort, sur chaque structure, résultant des pressions sur les parties immergées, serait facile à calculer.

Par suite des réflexions partielles sur les parois et de la diffraction, l'écoulement réel diffère de la 
houle à l'infini, appelée encore «houle incidente». L'agitation peut présenter des valeurs élevées par concentrations en certains points des ondes réfléchies. Les efforts réels vont également dépendre de la perturbation causée par les obstacles.

Deux résultats sont donc intéressants dans l'étude la diffraction de la houle :

- l'agitation, ou la surface libre au voisinage de l'obstacle et sur sa paroi afin de pouvoir définir les conditions d'aceès et les conditions d'amarrage;

- les efforts et moment résultant des pressions du fluide sur l'obstacle.

\section{* *}

La houle incidente considérée est la houle de Stokes limitée au premier ordre. Toutes les grandeurs de l'écoulement sont donc ramenées au paramètre $\varepsilon$ caractérisant la perturbation par rapport à l'état de repos. La cambrure, c'est-à-dire le rapport $\varepsilon$ de l'amplitude $h$ à la longueur d'onde $\lambda$, est supposée petite et l'on négligera dans les calculs les termes d'ordre supérieur à $\varepsilon$.

On suppose que la houle incidente est établie depuis un temps suffisamment long pour donner lieu à un écoulement stationnaire.

L'écoulement du fluide supposé parfait est donc défini par son potentiel $\Phi=\mathcal{K}\left\{e^{i \sim t} \varphi\right\}$. Toutes les équations du problème étant linéaires, on recherchera le potentiel $\varphi$ sous la forme $\varphi=\varphi_{d}+\varphi_{\infty}, \varphi_{\infty}$ étant le potentiel de la houle incidente et $\varphi_{d}$ le potentiel correctif dû à la présence des obstacles.

Cela revient donc à considérer l'écoulement résultant comme la superposition de l'écoulement incident et de l'écoulement perturbé dù à l'opacité des obstacles.

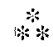

Les théries des efforts sur les structures placées dans un fluide en mouvement distinguent en général deux composantes à la force agissant sur un élément de surface. L'une, proportionnelle à l'accélération du fluide en l'absence d'obstacle, tient compte des effets d'inertie (et de gravité) transmis par le fluide; l'autre, qui est proportionnelle au carré de la vitesse du fluide, représente la résistance de forme de l'obstacle et la résistance de frottement du fluide sur les parois.

Seule la première composante est considérée ici puisque le fluide est supposé parfait et que l'écoulement est potentiel.

La pression permettant de calculer l'effort est domnée par l'équation de Bernoulli :

$$
\frac{p}{p}=-g z-\frac{\partial \Phi}{\partial t}
$$

On intégrera $p$ du fond à la surface de repos du fluide $z=0$, la contribution du fluide située entre la surface libre $\eta$ et $z=0$ étant du second ordre. Le terme - $g z$ représente une pression uniforme dans une tranche horizontale; l'effort résultant de cette pression sera donc nul, de sorte qu'il nous suffira de calculer :

$$
\frac{p}{\rho}=-\frac{\partial \Phi}{\partial t}
$$

soit :

$$
\frac{p}{\rho}=\mathcal{R}\left\{-i \sigma\left(\varphi_{\infty}+\varphi_{d}\right) e^{i \sigma t}\right\}
$$

\section{Les équations en théorie linéaire}

Le problème considéré est celui de l'écoulement irrotationnel d'un fluide incompressible autour d'obstacles fixes, Ie fluide s'étendant dans toutes les directions horizontales jusqu'à l'infini.

Avant l'introduction des obstacles, le fluide est soumis à une houle cylindrique monochromatique définie par sa période $\mathrm{T}$, son incidence $\theta$ et son amplitude $h$.

Le potentiel $\Phi_{\infty}$ d'une telle houle, si $d$ est la profondeur, est :

$$
\begin{gathered}
\Phi_{\infty}=\frac{g h}{\sigma} \frac{\operatorname{ch~} m_{0}(z+d)}{\operatorname{ch~} m_{0} d} e^{i\left(\sigma t-m_{0} x \cos \theta-m_{0} y \sin \theta\right)} \\
m_{0}=\frac{2 \pi}{\lambda} \quad m_{0} \text { th } m_{0} d=\frac{\sigma^{2}}{g}
\end{gathered}
$$

Le champ des vitesses dues à la présence des obstacles étant supposé irrotationnel, on le définit par le potentiel inconnu $\Phi_{d}$.

$\Phi_{d}$ doit satisfaire à trois types de conditions :

- il doit vérifier l'équation de Laplace dans le domaine extérieur aux obstacles (région D);

- il doit avoir une dérivée normale donnée sur la surface des obstacles;

- il doit satisfaire aux conditions aux limites du domaine fluide : condition de fond, condition de surface libre et condition de radiation a l'infini dans le plan horizontal.

Ces conditions s'écrivent en posant:

$$
\begin{gathered}
\Phi_{d}=e^{i s l} \varphi_{d}(x, y, z) \\
\Delta \varphi_{d}=0 \text { dans } \mathrm{D} \\
\left.\frac{d \varphi_{d}}{d n}\right|_{s}=-\left.\frac{d \varphi_{\infty}}{d n}\right|_{s} \text { opacité des parois } \\
\left.\frac{\partial \varphi_{d}}{\partial z}\right|_{z=-a}=0 \text { opacité du fond } \\
\frac{\partial \varphi_{d}}{\partial z}+\frac{\sigma^{2}}{g} \varphi_{d}=0 \quad \text { surface libre } \\
\lim _{r \rightarrow \infty} \sqrt{r}\left(\frac{\partial \varphi_{d}}{\partial r}+i \mathrm{~K} \varphi_{d}\right)=0 \quad \text { si } r \rightarrow \infty, \quad K=\frac{\sigma^{2}}{g}
\end{gathered}
$$

Cette dernière condition exprime que $\varphi_{d}$ se comporte à l'infini comme une onde plane divergente issue des obstacles placés dans l'écoulement.

\section{REPRÉSENTATION DE LA SOLUTION PAR UN POTENTIEL DE SIMPLE COUCHE.}

Suivant une méthode générale en écoulement potentiel, on représente les obstacles par une distribution de sources réparties sur leurs surfaces immergées (S). $\varphi_{d}$ est défini par la densité de la distribution qui produit sur (S) une vitesse normale donnée par la condition d'opacité des parois. 


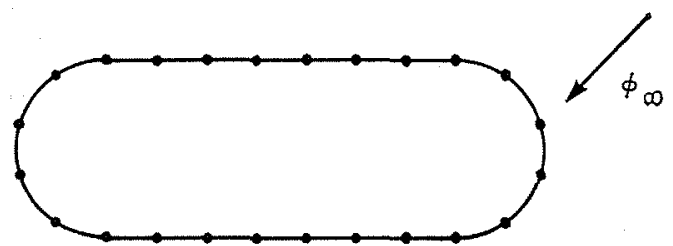

1/ Distribution de sources.

Source distribution.

Soit G (M, P) le potentiel en M d'une source placée en P. G (M, P) satisfait à la condition (1) sauf en $P$ où il comporte une singularité en $1 /[R(M, P)]$, et aux conditions (3), (4) et (5). Il s'écril donc :

$$
\mathrm{G}(\mathrm{M}, \mathrm{P})=\frac{1}{\mathrm{R}(\mathrm{M}, \mathrm{P})}+\mathrm{W}(\mathrm{M}, \mathrm{P})
$$

Le potentiel dù à la distribution de sources, de densité $\tau(P)$, réparties sur $(\mathrm{S})$ est :

$$
\varphi_{d l}(\mathrm{M})=\iint_{s} \mathrm{G}(\mathrm{M}, \mathrm{P}) \tau(\mathrm{P}) d \mathrm{~S}
$$

La densité $\tau(\mathrm{P})$ est définie par la condition d'opacité des parois. Lorsque $\mathrm{M} \rightarrow \mathrm{P}$ la dérivéc normale du potentiel de simple couche subit une discontinuité égale à $-2 \pi \tau(\mathrm{P})$ :

$$
\frac{d \varphi_{d}}{d n_{\mathrm{M}}}=-2 \pi \tau(\mathrm{P})+\frac{d \varphi_{d}}{d n_{\mathrm{P}}}
$$

soit :

$$
-2 \pi \tau(P)+\iint_{s} \frac{\partial}{\partial n_{1}} G(P, \mu) \cdot \tau(\mu) d s_{\mu}
$$

$n_{\mathrm{P}}$ étant la normale en $\mathrm{P}$ extérieure aux obstacles.

La détermination de la densité $\tau(\mathrm{P})$ se ramène donc à la résolution d'une équation intégrale de Fredholm de deuxième espéce. John (1950) a montré l'existence et l'unicité de la solution, même dans le cas où le noyau de l'intégrale devient singulier.

La méthode précédente n’impose aucune condition quant à la forme des obstacles. Elle suppose simplement que la normale à la surface (S) est continue. Cette restriction peut être levée facilement en remplaçant tous les angles des parois par des arrondis de faible rayon.

Le noyau de l'intégrale de Fredholm est défini par la fonction de Green du problème $G(M, P)$. En profondeur constante $d, \mathrm{G}(\mathrm{M}, \mathrm{P})$ est donnée par :

$$
\begin{aligned}
& \mathrm{G}(\mathrm{M}, \mathrm{P})=\frac{1}{\mathrm{R}}+\frac{1}{\mathrm{R}^{\prime}}+ \\
& \int_{0}^{\infty} \frac{2(\mathrm{~K}+\mu) e^{-\mu l} \operatorname{ch} \mu(z+d) \operatorname{ch} \mu(\zeta+d)}{\mu \operatorname{sh} \mu d-\mathrm{K} \operatorname{ch} \mu d} \mathrm{~J}_{0}(\mu r) d \mu \\
& \mathrm{R}=\left[(x-\xi)^{2}+(y-\eta)^{2}+(z-\zeta)^{2}\right]^{1 / 2} \\
& \mathrm{R}^{\prime}=\left[(x-\xi)^{2}+(y-\eta)^{2}+(z+\zeta)^{2}\right]^{1 / 2} \\
& r=\left[(x-\xi)^{2}+(y-\eta)^{2}\right]^{1 / 2} \\
& \mathrm{M}(x, y, z)
\end{aligned}
$$

John (1950) a donné à partir de cette expression un développement en série de fonctions orthogonales :

$$
\begin{gathered}
\mathrm{G}(\mathrm{M}, \mathrm{P})=-2 i \pi \sum_{i=0}^{\infty} \times \\
\times \mathrm{C}_{i} \mathrm{H}_{0}{ }^{(2)}\left(m_{i} l^{2}\right) \operatorname{ch} m_{i}(z+d) \operatorname{ch~} m_{i}(\zeta+d) \\
\mathrm{C}_{i}=\frac{m_{i}{ }^{2}-k^{2}}{d\left(m_{i}^{2}-k^{2}\right)+\mathrm{K}}
\end{gathered}
$$

$m_{i}$ th $m_{i} d=\mathrm{K}$

Un développement analogue pour $\tau(\mathrm{P})$ conduit à poser :

$$
\tau(\mathrm{P})=\sum_{i=0}^{\infty} \mathrm{C}_{i}^{\prime} \operatorname{ch} m_{i}(z+d) \alpha_{i}(x, y) \quad \mathrm{P}(x, y, z)
$$

Le second membre de l'intégrale de Fredholm, $d \Phi_{o} / d n_{\mathrm{P}}$ ne comporte que le premier terme de la série en ch $m_{i}(z+d)$, on prendra donc :

$$
\tau(\mathbf{P})=\frac{\operatorname{ch} m_{0}(z+d)}{\operatorname{ch} m_{0} d} \alpha(x, y)
$$

Le problème se ramène ainsi, comme on pouvait le prévoir à un problème à deux dimensions. La fonction inconnue $\alpha(x, y)$ vérifie l'équation intégrale :

$$
2 \pi \alpha(\mathrm{P})+i \pi \int_{\mathrm{c}} \frac{\partial}{\partial n_{\mathrm{P}}} \mathrm{H}_{0}{ }^{(2)}\left(m_{0} r_{\mu \mathrm{P}}\right) \cdot \alpha(\mu) d \mathrm{C}_{\mu}=\left.\frac{d \varphi_{\infty}}{d n_{\mathrm{P}}}\right|_{z=0}
$$

(C) contour horizontal des obstacles.

La solution $\varphi_{d}$ s'en déduit par :

$$
\varphi_{d}=-i \pi \frac{\operatorname{ch} m_{0}(z+d)}{\operatorname{ch} m_{0} d} \int_{\mathrm{C}} \alpha(\mu) \mathrm{H}_{0}{ }^{(2)}\left(m_{0} r_{\mu \mathrm{P}}\right) d \mathrm{C}_{\mu}
$$

Si $d \rightarrow \infty$ on obtient le cas d'une profondeur infinie pour lequel la densité de sources est :

$$
\tau(\mathrm{P})=e^{k x} \alpha(x, y)
$$

\section{SURFACE LIBRE ET EFFORTS :}

La surface libre $\eta$ est la partie réelle

$$
\mathcal{\alpha}\left\{-\frac{i \sigma}{g} e^{i \sigma t} \varphi(x, y, 0)\right\}
$$

et l'effort s'en déduit par la relation simple :

$$
\overrightarrow{\mathrm{R}}=-\int_{0} \overrightarrow{p n d} \mathrm{~S}=-\rho g \frac{\operatorname{th} m_{0} d}{m_{0}} \int_{\mathrm{C}} \eta \vec{n} d \mathrm{C}
$$

de résolution Méthode

La partie essentielle du calcul se ramène donc à la résolution numérique de l'équation intégrale de Fredholm. Dans ce but on discrétise les équations en remplaçant la surface réelle des obstacles par une surface approchée composée de «palplanches» dont on choisira le nombre en fonction de la précision demandée. Le contour (C) de chaque obstacle 


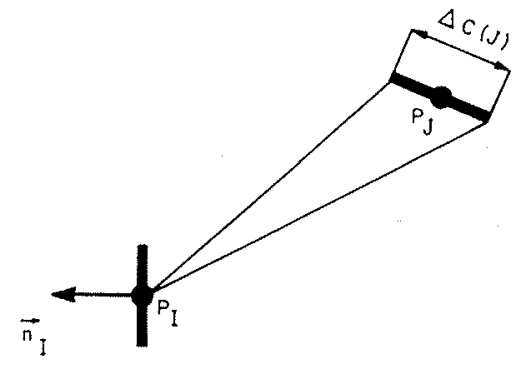

2/ Point de contrôle. Control point.

est remplacé par un contour polygonal. Sur chaque côté, la fonction $\alpha(x, y)$ est constante.

L'équation intégrale se ramène ainsi à un système d'équations linéaires en nombre égal au nombre $\mathrm{N}$ des palplanches. Les inconnues étant les valeurs de la fonction $\alpha(x, y)$ sur chaque palplanche : soit $\alpha_{i}, i$ variant de $1 \dot{a} \mathrm{~N}$.

La matrice des coefficients des $\alpha_{i}$ comporte $2 \pi$ sur toute sa diagonale principale. Les autres coefficients sont de la forme :

$$
A_{i j}=i \pi \int_{\Delta \mathrm{C}_{j}} \mathrm{H}_{0}^{(2)}\left(m_{0} r_{\mathrm{P}_{i} \mathrm{P}_{j}}\right) d \mathrm{C}
$$

$P_{i}$ étant le point où l'on écrit la condition d'opacité et $P_{j}$ le point courant sur le segment $\Delta C_{j}$ (fig. 2).

L'intégrale porte uniquement sur des éléments géométriques connus. On pourra soit la calculer numériquement, soit appliquer une formule simple d’approximation si la précision le permet.

\section{Précision des résultats}

Le calcul numérique précédent fait intervenir la précision en trois points : le choix du nombre de palplanches, de largeur $\Delta \mathrm{C}_{i}$, le choix $\mathrm{du}$ point de contrôle sur chaque segment du contour polygonal et le calcul des coefficients de la matrice.

On appelle point de contrôle le point où l'on impose effectivement la condition :

$$
\frac{d \varphi_{\infty}}{d n}=-\frac{d \varphi_{d}}{d n}
$$

Il existe une assez grande liberté dans le choix de ce point. En général les résultats les meilleurs seront obtenus en prenant le centre géométrique du segment correspondant.

Le nombre de palplanches fixe le rang du système linéaire à résoudre. Un nombre $\mathrm{N}$ élevé augmentera le temps de calcul portant sur les matrices. Il permettra par contre souvent une économie de temps dans le calcul des coefficients $A_{i j}$. Ces coefficients peuvent en effet se calculer par la formule de la moyenne, sans quadrature, si le domaine d'intégration $2 \pi \Delta \mathrm{C} / \lambda$ est petit devant la distance entre les zéros des fonctions $\mathbf{J}_{0}$ et $Y_{0}$. Un optimum est à rechercher.

Le critère de précision est donc d'une part fonction du rapport de $\Delta \mathrm{C}$, à la dimension moyenne de l'obstacle - l'obstacle réel est défini d'autant mieux que ce rapport est faible - et d'autre part du rapport $\Delta C / \lambda$. On voit en particulier que si l'on dimi- nue trop la longueur d'onde $\lambda: \Delta C / \lambda>10^{-2}$, le calcul des coefficients $A_{i j}$ par intégration numérique, ou par quadrature si cela est possible, devient obligatoire.

Pratiquement, si $\mathrm{N}$ est inférieur à 150, on utilisera une méthode de résolution directe pour inverser le système linéaire.

Si $N$ est supérieur à 150 , le calcul nécessitera en général l'emploi de mémoires auxiliaires. On utilisera plutôt une méthode d'itération.

Pour un calculateur tel que IBM 7094, le temps de calcul est de l'ordre de $3 \mathrm{mn}$ pour un obstacle de 50 palplanches. Il peut atteindre $30 \mathrm{~mm}$ si le rapport $\Delta \mathrm{C} / \lambda$ est tel qu'il faille calculer avec précision les $A_{i j}$. Dans ce cas le calcul peut être réparti en quatre phases :

- calculs géométriques : distances entre points de contrôle, dérivée normale, etc.;

- calculs de la matrice $\mathrm{A}_{i j}$;

_ inversion du système;

- restitution de la surface libre et effort.

Les deux premières phases occupent à elles seules en général les deux tiers du temps de calcul.

Tout ceci suppose bien entendu que la surface libre est restituée seulement aux points de contrôle. Sinon il faut doubler ou tripler les temps précédents.

On peut constater sur les résultats figurant dans la deuxième partie que la précision est très bonne mème pour une schématisation assez approchée du contour réel des obstacles.

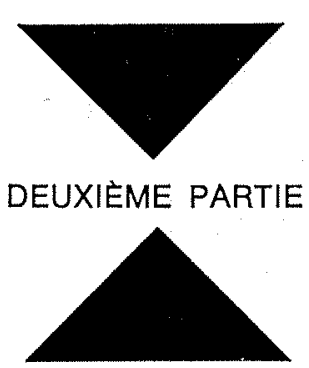

Introduction

Les programmes de calcul «Fortran» sont rédigés pour IBM 7094. Ils utilisent tous les sous-programmes d'opération matricielle contenus en bibliothèque. Pour les cas les plus volumineux (N supérieur à 50), la mémoire centrale n'était pas suffisante, il a fallu utiliser des tambours et des bandes magnétiques supplémentaires, et structurer le programme en «Overlay».

Suivant le problème étudié, les «sorties» sont très variables. Elles peuvent comporter l'agitation le long de la pile ou à quelque distance de la pile, l'effort maximal et son point d'application, les moments des couples horizontaux et verticaux, leur déphasage, etc.

Les temps de calcul dépendent naturellement du rang $\mathrm{N}$ du système et des « sorties » demandées.

Pour une pile de 50 palplanches, la restitufion de la surface libre le long de la pile et les calculs d'effort ont demandé de 3 à $10 \mathrm{mn}$ environ. 


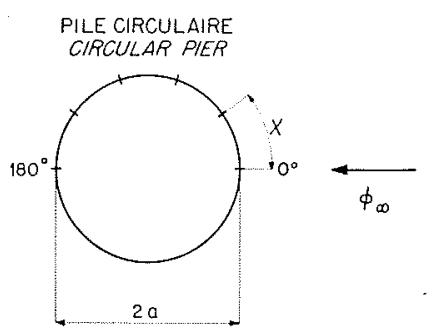

PILE CYLINDRIQUE (I) CYLINORICAL PIER (I)
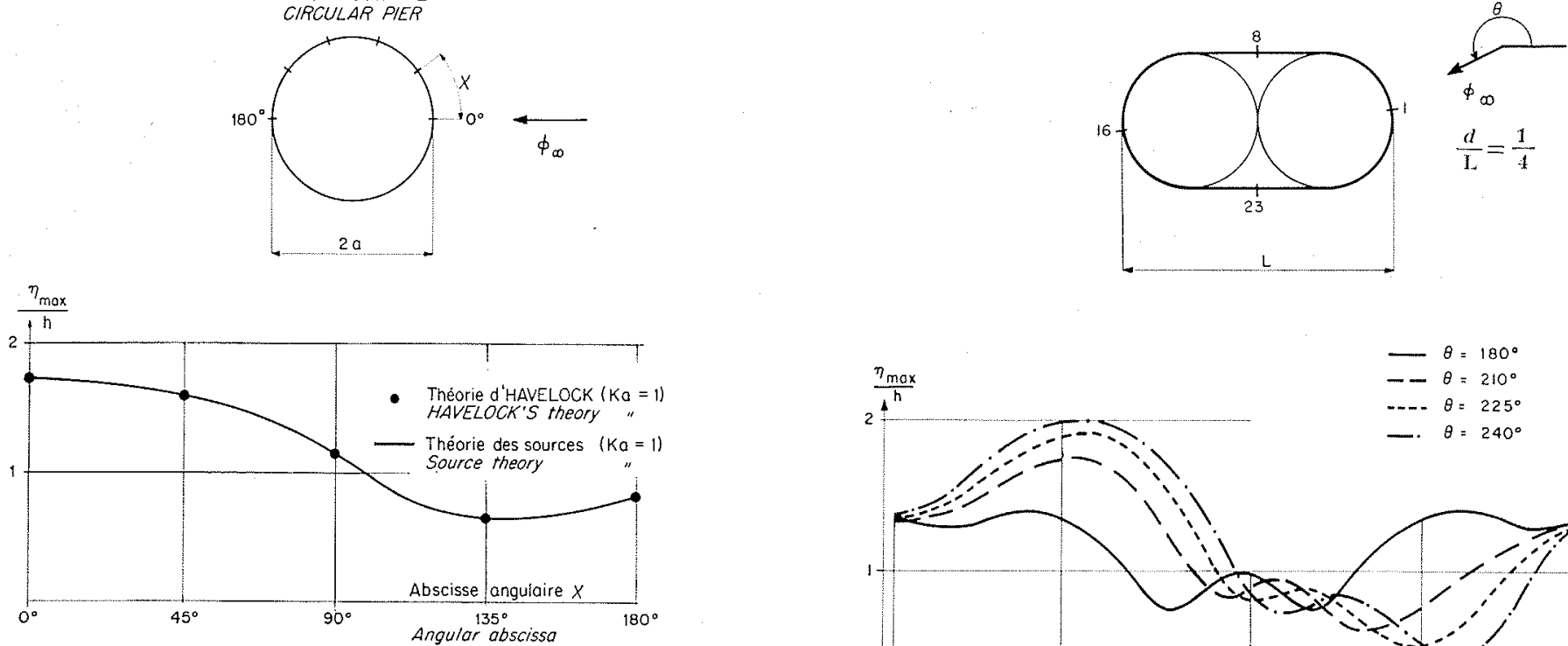

3/ Agitation maximale $\left(\eta_{\max } / h\right)$ le long du cylindre.

Maximum wave $\left(\eta_{m a x} / h\right)$ along the oylinder.

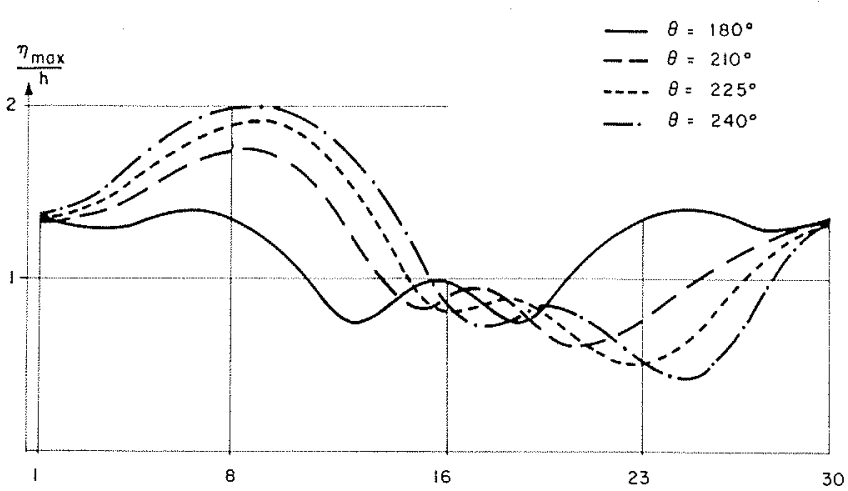

4/ Agitation le long de la pile cylindrique $1.7 . / \mathrm{L}=2,5$.

Waves along cylindrical pier $(1), \lambda / L=2.5$.

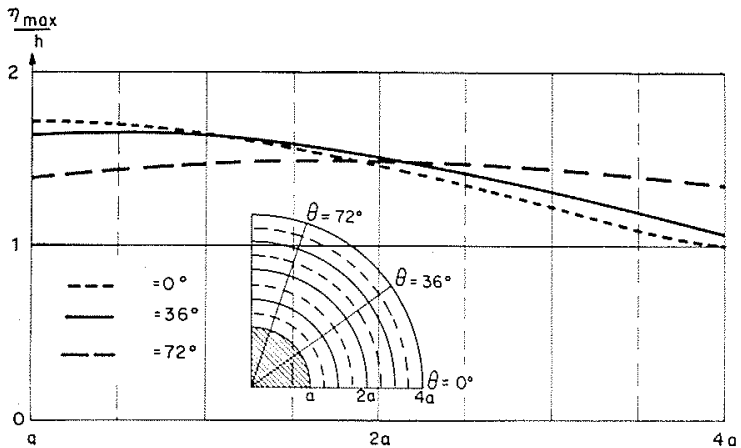

3 bis/ Agitation au voisinage du cylindre $\left(m_{v}(t=1)\right.$.

Waves near the cylinder $\left(\mathrm{m}_{\theta} \mathrm{a}=1\right)$.

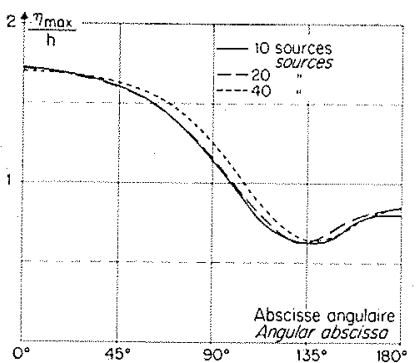

3 ter/ Agitation maximale pour un cercle défini par 10, 20 et 40 sources $\left(m_{0} a=1\right)$

Maximum waves for a given circle, as produced by 10 , 20 and 40 sources $\left(\mathrm{m}_{i} \mathrm{a}=1\right)$.

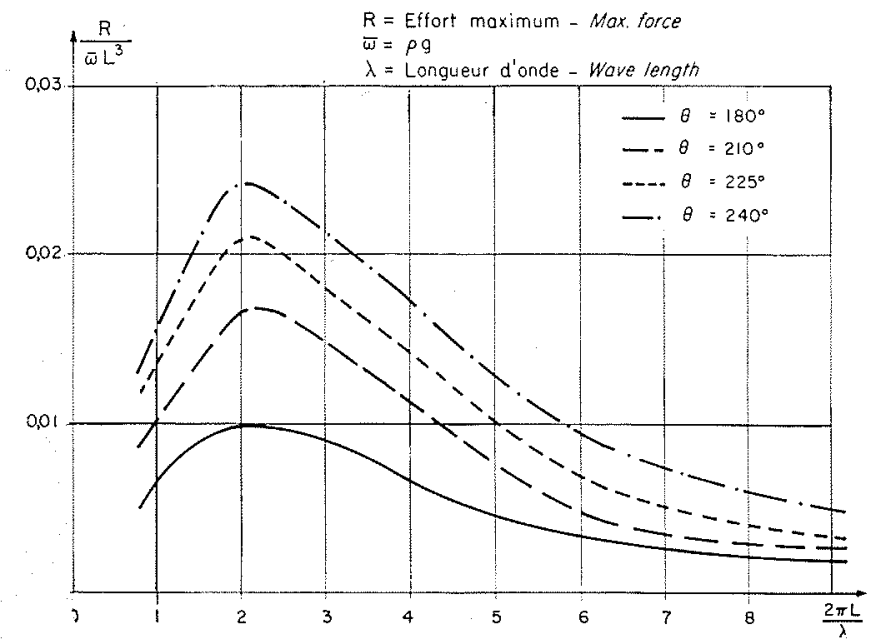

4 bis/ Effort maximal $\mathrm{R}$ en fonction de $2 \pi \mathrm{L} / \lambda(h=1)$. Pile 1 Maximum force $\mathrm{R}$ against $2 \pi L / \lambda(h=1)$. Pier (1).

Applications

Nous donnons pour finir quelques résultats graphiques parmi les calculs effectués pour différentes sortes de piles.

Les figures (3) montrent la concordance entre la solution analytique (Havelock) et la méthode des sources.

La figure 3 ter donne la hauteur maximale de houle le long du cylindre pour $\mathrm{N}=10,20,40$. (Hauteur rapportée à celle de la houle à l'infini.)

Les figures 4 à 8 domnent la hauteur mouillée et l'effort pour différentes formes de piles et différentes valeurs de l'angle d'incidence de la houle. Il s'agit toujours des valeurs maximales $\eta_{\max }$ et $R_{\max }$. 


\section{A. DAUBERT et J.-C. LEBRETON}

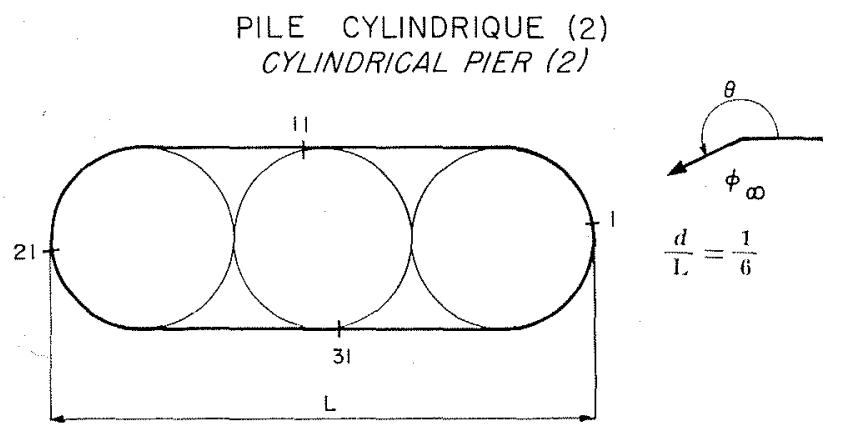

PILE ELLIPTIQUE (3)

ELLIPTICAL PIER (3)
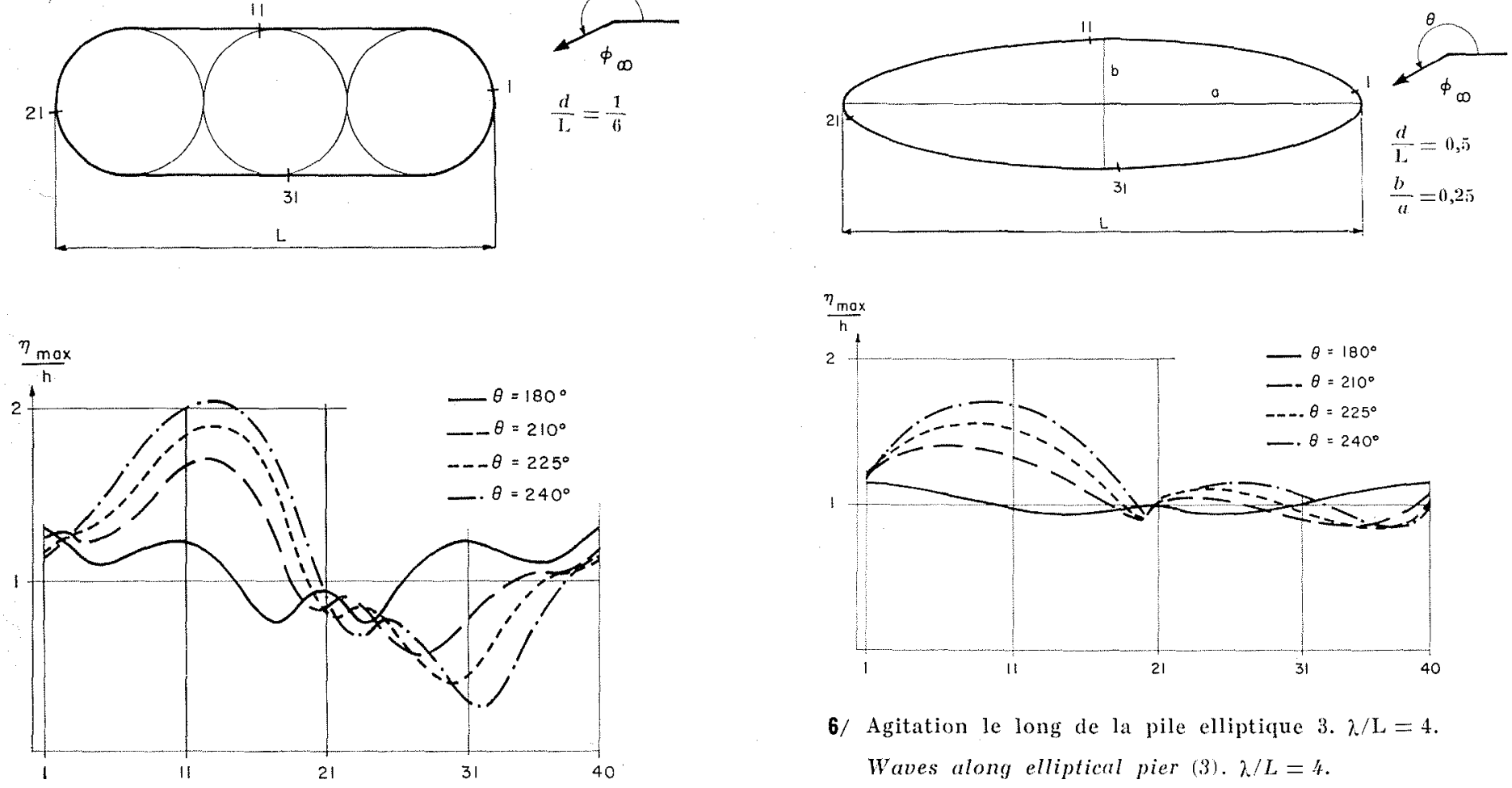

6/ Agitation le long de la pile elliptique $3 . \lambda / L=4$.

Waves along elliptical pier (3). $\lambda / L=4$.

5/ Agitation le long de la pile eylindrique 2. $\lambda / \mathrm{L}=1,67$.

Waves along eylindrical pier $(2) . \lambda / L=1.67$.

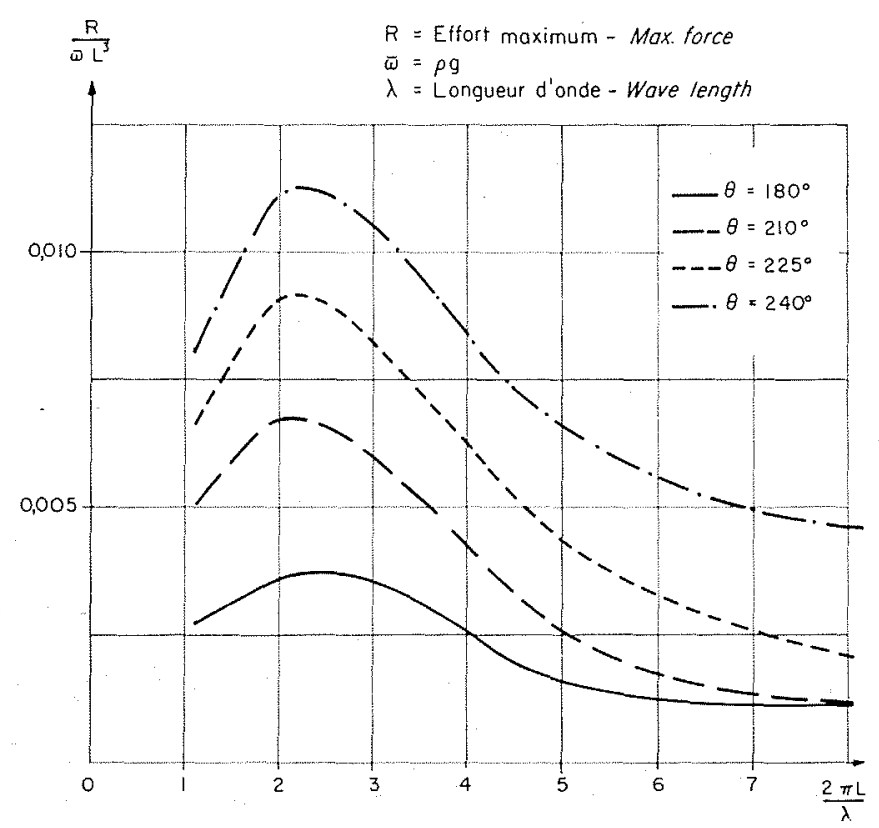

5 bis/ Effort maximal $\mathrm{R}$ en fonction de $2 \pi \mathrm{L} / \lambda(h=1)$. Pile 2. Maximum force $R$ against $2 \pi L / \lambda(\mathrm{h}=1)$. Pier (2).

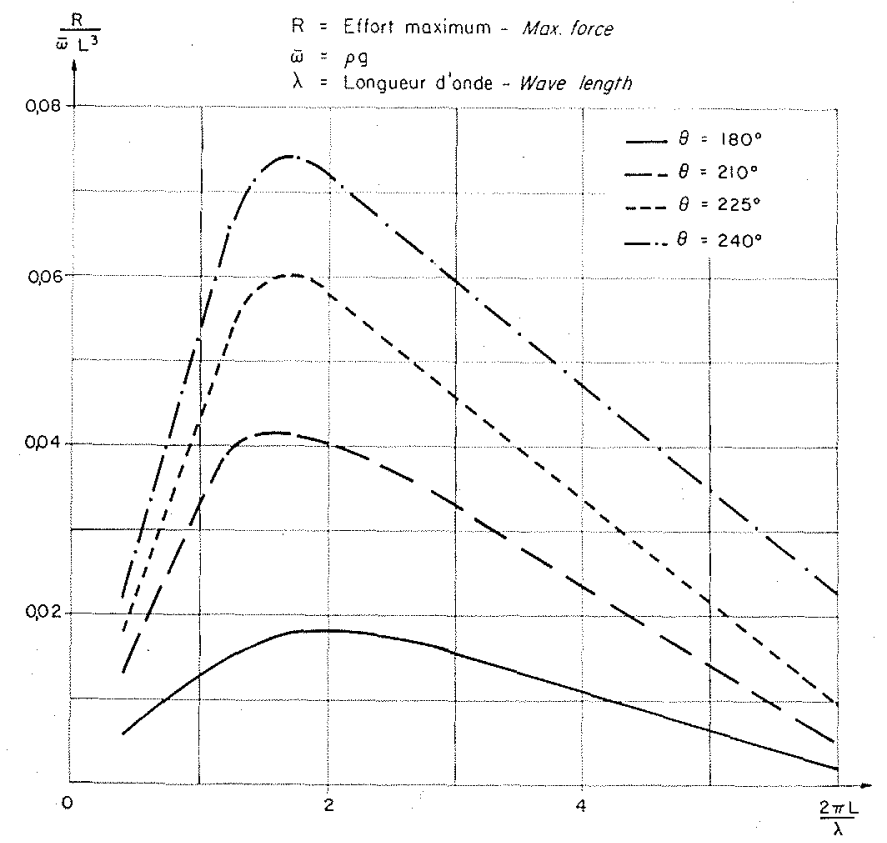

6 bis/ Effort maximal $\mathrm{R}$ en fonction de $2 \pi \mathrm{L} / \lambda(h=1)$. Pile 3 . Maximum force $R$ against $2 \pi L / \lambda(\mathrm{h}=1)$. Pier (3).
$* *$

En conclusion, la théorie d'Havelock donne une solution élégante et directe pour des corps minces (rapport de la largeur à la longueur d'onde petit) et la méthode des sources réparties sur la surface vient la compléter, lorsque l'épaisseur des obstacles n'est plus négligeable devant la longueur d'onde.

Signalons que des programmes analogues ont été établis pour l'étude des efforts sur les carènes des 
PILE A DIEDRES (5)

DIHEORON - ENDED PIER (5)

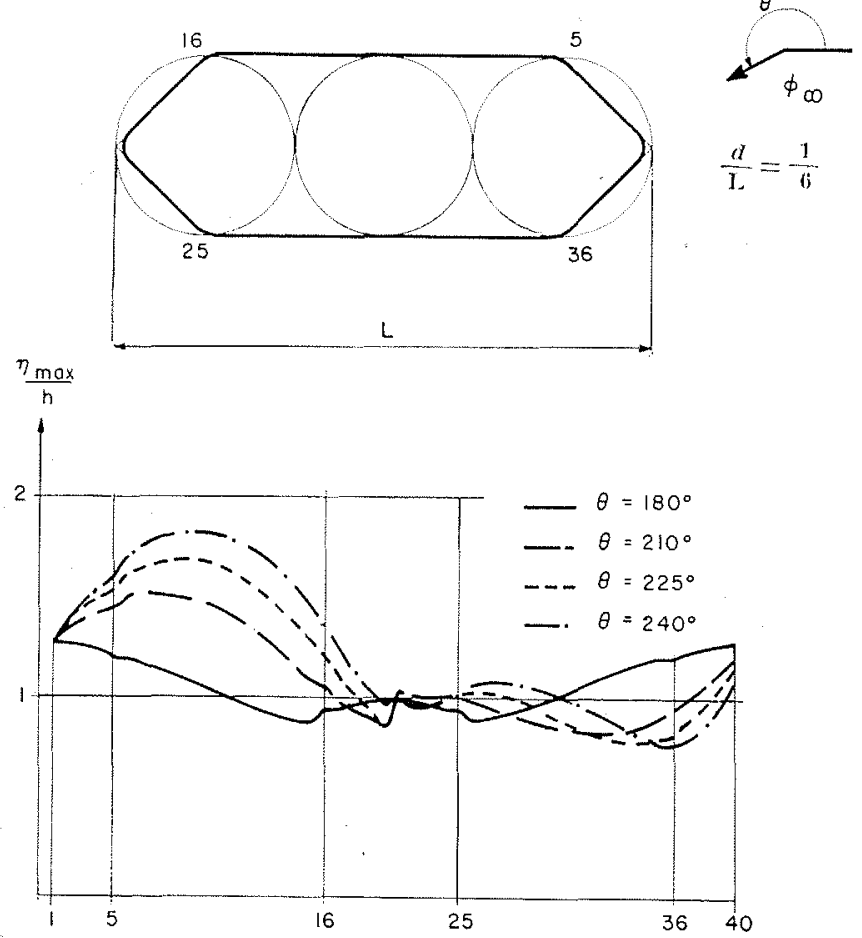

1/ Agitation le long de la pile à dièdres $5 . \lambda / \mathrm{L}=3,3$. Waves along dihedron-ended pier (5). $\lambda / L=3.3$.

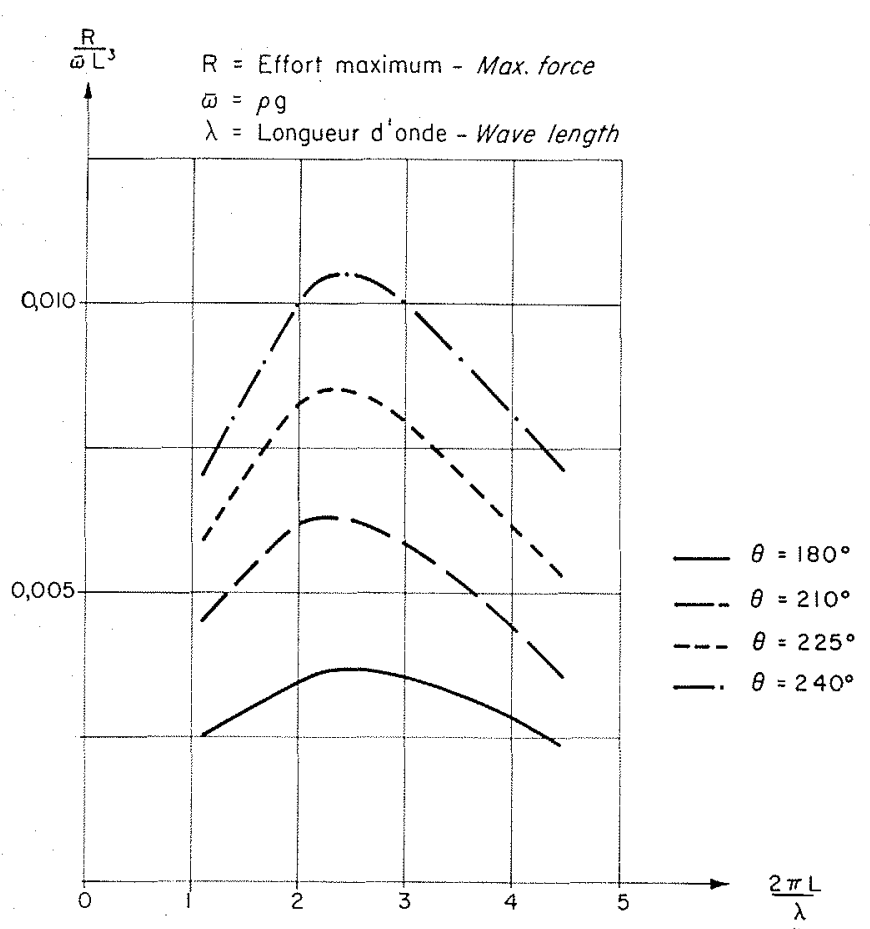

7 bis/ Effort maximal $R$ en fonction de $2 \pi L / \lambda(h=1)$. Pile 5 . Maximum force $R$ against $2 \pi L / \lambda(\mathrm{h}=1)$. Pier $(5)$.

navires et pour l'étude de la diffraction dans les ports.

Nous remercions M. Caseau pour les conseils qu'il nous a donnés au cours de cette étude ainsi que Mlle Margnac qui a bien voulu se charger de la partie numérique.
CYLINDRICAL PIERS (4)

PILES CYLINDRIQUES (4)
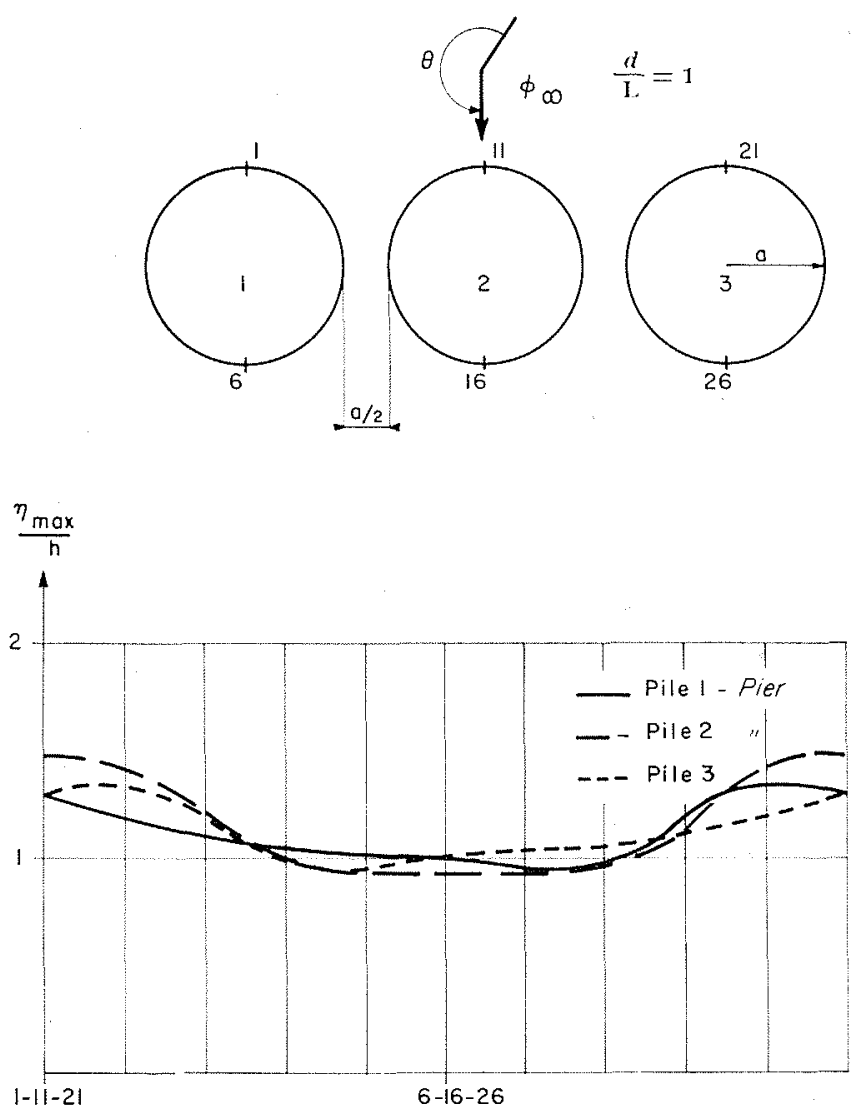

8/ Agitation autour de trois piles cylindriques $4 . \lambda / a=10$. Waves around three cylindrical piles (4). $\lambda / \mathrm{a}=10$.

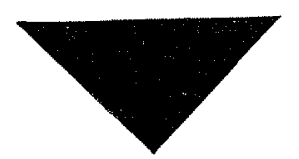

Références

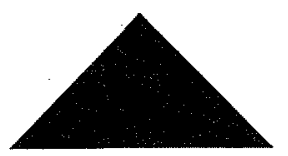

Covrant et Hrbert. - Vol. I et II. Methods of Mathematical Physics. Interscience Publishers, 1962.

KELLOG (O. D.). - Foundations of potential theory. J. Springer, Berlin, 1929.

John (F.). - On the motion of floating bodies. Communications of Pure and Applied Mathematics (1950).

Hess and S.rTh. - Calculation of potential flow about arbitrary body shape. Douglas Aircraft Division, paper 1700 .

Knx. - On the harmonic oscillations of a rigid body on a free surface. Flaids Mechanics (1965), vol. 21, p. 3.

Bonnefille et Germain. - Action de la houle sur des ouvrages isolés de grandes dimensions. A.I.R.H., paper, 1, 40. Congrès de Londres 1963.

Havelock. - The pressure of water waves upon a fixed obstacles. Proceedings of the Royal Society of London, A. $175,1940$. 


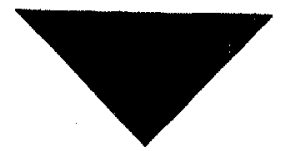

Abstract

Wave diffraction on vertical-sided piers

by

A. Daubert * and J.-C. Lebreton *

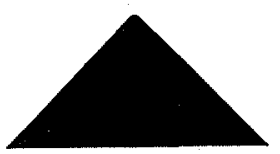

It is proposed to calculate in a linear irrotational wave theory the forces acting on vertical-sided structures subjected to waves coming in from an infinite distance.

The problem is solved by determining the diffracting wave potential and adding it to the incident waves, which is done in two phases, as follows:

1) Finding Green's function (Eqs. 1 to 5);

2) Determining the intensity of the sources to distribute over the obstacle.

Green's "influence" function represents the singular potential created by a source. Source intensity is determined by expressing obstacle wall impermeability, which leads to a Fredholm integral equation. A numerical solution method for this equation has been applied with an IBM 7094 computer. The results were first compared with Havelock's analytical solution for a circular pier (Fig. 3), which confirmed the accuracy of the numerical solution.

Figures 4 and 5 show results for piers formed by envelopes of two and three circles respectively. Figures 6 and 7 refer to elliptical and dihedron-ended piers, and Figure 8 is an example with obstacles in the form of three separate circular piers.

* Engineers at the Centre de Recherches et d'Essais de Chatou (E.D.F.).

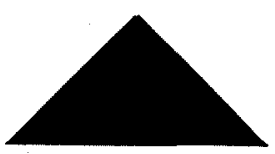

